\title{
Probing charged lepton flavor violation with the Mu2e experiment
}

\author{
S. E. Müller, A. Ferrari for the Mu2e-collaboration
}

Helmholtz-Zentrum Dresden-Rossendorf

DPG Spring Meeting, München, March 22, 2019

This document was prepared by Mu2e collaboration using the resources of the Fermi National Accelerator Laboratory (Fermilab), a U.S. Department of Energy, Office of Science, HEP User Facility. Fermilab is managed by Fermi Research Alliance, LLC (FRA), acting under Contract No. DE-AC02-07CH11359.

חבק
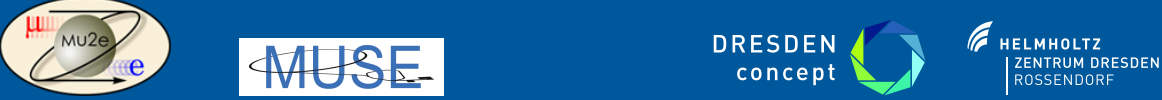


\section{Motivation}

The Standard Model of particle physics currently contains:

- Quark mixing

- Transitions between charged and neutral leptons of same flavor

- Neutrino oscillations

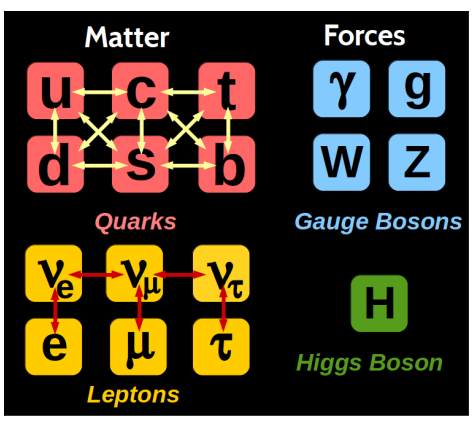




\section{Motivation}

The Standard Model of particle physics currently contains:

- Quark mixing

- Transitions between charged and neutral leptons of same flavor

- Neutrino oscillations

No charged lepton flavor violation (CLFV) observed so far!

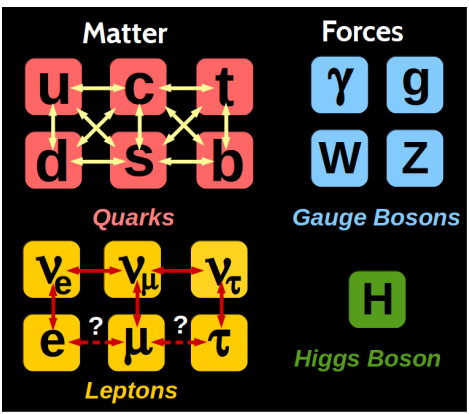




\section{Motivation}

The Standard Model of particle physics currently contains:

- Quark mixing

- Transitions between charged and neutral leptons of same flavor

- Neutrino oscillations

No charged lepton flavor violation (CLFV) observed so far!

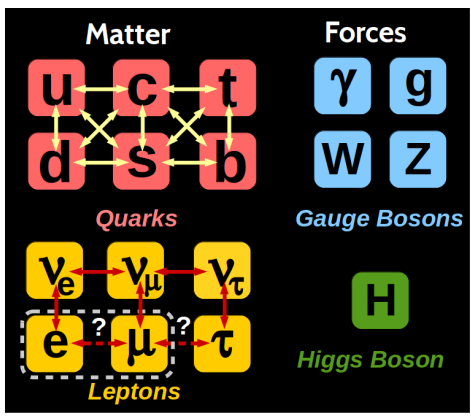

Mu2e will search for the neutrinoless conversion of a muon into an electron in the coulomb field of a nucleus $(\mu \mathrm{N} \rightarrow \mathrm{eN}$ ) with a projected upper limit of $8 \times 10^{-17}(90 \% \mathrm{CL})$

Current limit by SINDRUM-II (PSI): $\mathrm{B}(\mu \mathrm{Au} \rightarrow \mathrm{eAu})<7 \times 10^{-13}(90 \% \mathrm{CL})$

SM prediction via neutrino mixing is $\sim 10^{-54}$, but extensions of SM predict values up to $\sim 10^{-14}$ (Leptoquarks, heavy neutrinos, SUSY,...)

$\Rightarrow$ Unique possibility to test for New Physics 


\section{New physics}

Model independent Lagrangian:

$$
L_{C L F V}=\underbrace{\frac{m_{\mu}}{(\kappa+1) \Lambda^{2}} \bar{\mu}_{R} \sigma_{\mu \nu} e_{L} F^{\mu \nu}}_{\text {"Loop term" }}+\underbrace{\frac{\kappa}{(\kappa+1) \Lambda^{2}} \bar{\mu}_{L} \gamma_{\mu} e_{L}\left(\bar{e} \gamma^{\mu} e\right)}_{\text {"Contact term" }}
$$

$\Lambda$ : effective mass scale of New Physics $\kappa$ : relative contribution of contact term

Loop term: dominates for $\kappa \ll 1$

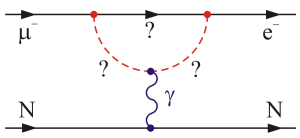

Contact term: dominates for $\kappa \gg 1$

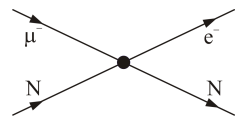

$5 \times 10^{4}$

all limits@90\% CL

$\mathrm{CR}(\mu \mathrm{N} \rightarrow e \mathrm{~N}$ on $\mathrm{Al})<6 \times 10^{-18}$

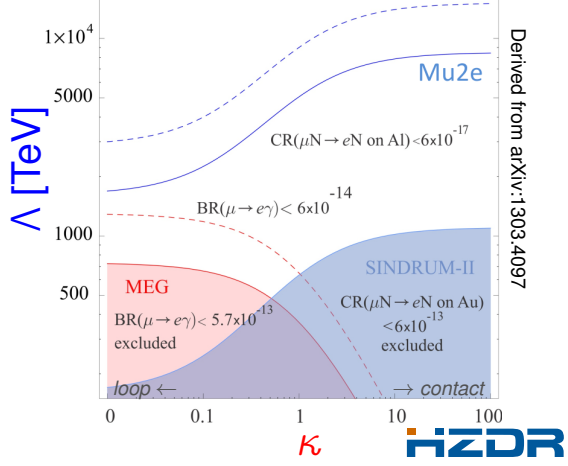

Mu2e will probe $\wedge \sim \mathrm{O}\left(10^{3}-10^{4}\right) \mathrm{TeV}$ 


\section{The Mu2e experiment}

The Mu2e experiment will search for CLFV in the process $\left(\mu^{-}+\mathrm{Al} \rightarrow \mathrm{e}^{-}+\mathrm{Al}\right)$

Stopped muons have a lifetime of $\sim 900$ ns in the 1 s orbital of the Al nucleus

- about $60 \%$ of stopped muons undergo the muon capture reaction $\left(\mu^{-}+{ }^{27} \mathrm{Al} \rightarrow \nu_{\mu}+{ }^{27} \mathrm{Mg}\right)$

- $40 \%$ of stopped muons decay in orbit (DIO)

- Michel spectrum of decay electrons stops around $\mathrm{M}_{\mu} / 2$

- CLFV signal for $\mu \rightarrow$ e conversion gives single mono-energetic electron

- $\mathrm{E}_{\mathrm{e}}=104.973 \mathrm{MeV} \simeq \mathrm{M}_{\mu}$

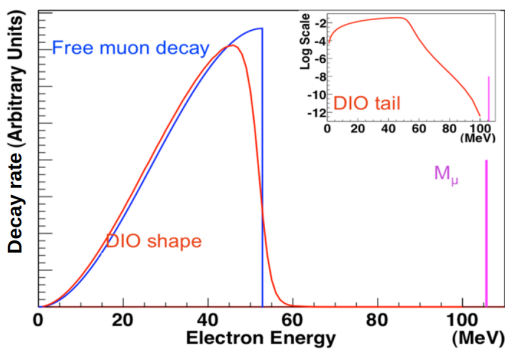

Normalized ratio $\mathrm{R}_{\mu \mathrm{e}}=\frac{\mathrm{N}\left(\mu^{-}+\mathrm{Al} \rightarrow \mathrm{e}^{-}+\mathrm{Al}\right)}{\mathrm{N}\left(\mu^{-}+\mathrm{Al} \rightarrow \text { nuclear capture }\right)}$ 


\section{The Mu2e experiment}

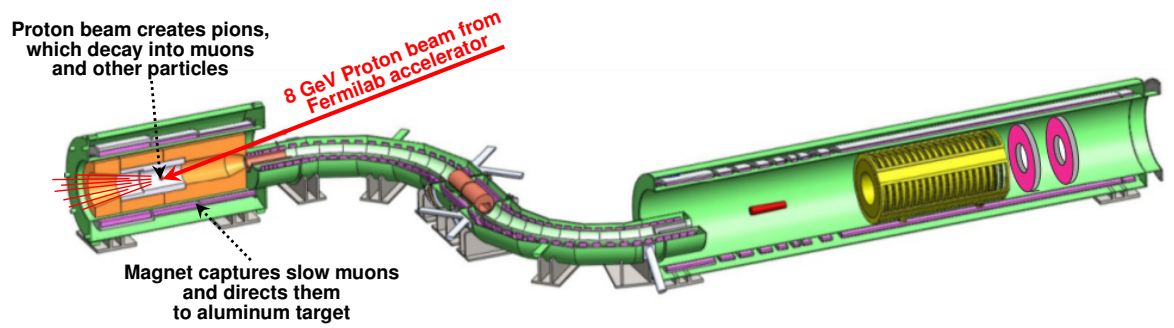

- Muons are produced by $8 \mathrm{GeV}$ proton beam on tungsten target

- time-averaged beam power: $7.3 \mathrm{~kW}$

- $4 \times 10^{7}$ protons/pulse, pulse separation: $1.695 \mu \mathrm{s}$

- Magnetic field in Production Solenoid guides produced pions towards Transport Solenoid

- Pions decay into muons 


\section{The Mu2e experiment}

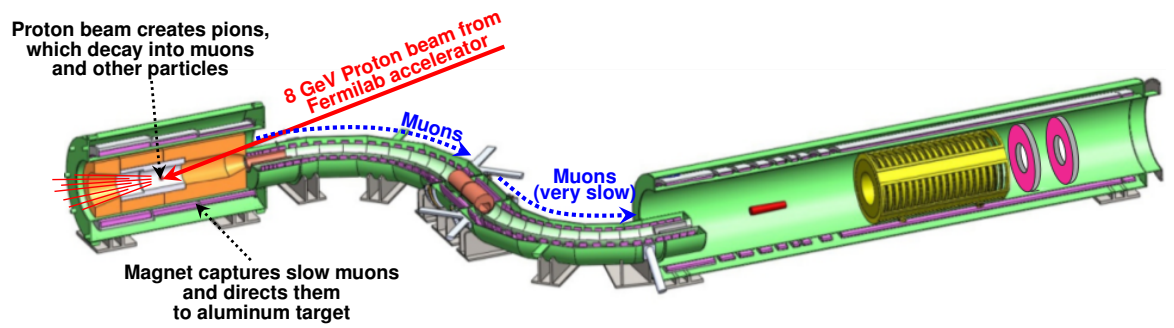

- Muons are transported in s-shaped Transport Solenoid

- Absorber foils remove antiprotons

- Solenoidal magnetic fields separate oppositely charged particles

- Collimators select low-momentum negatively-charged muons. 


\section{The Mu2e experiment}

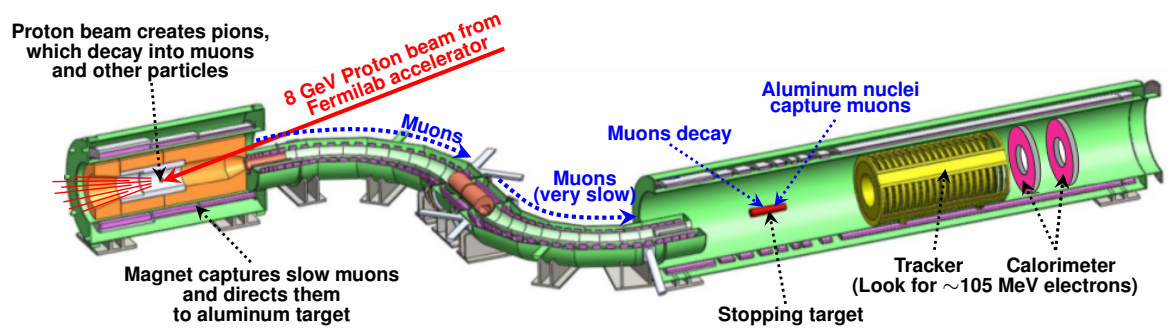

- Muons are stopped on aluminum target foils in Detector Solenoid

- stopped muons decay in orbit or are captured by the Al nucleus

- decay electrons are detected by a tracking detector and a calorimeter 


\section{The Mu2e experiment}

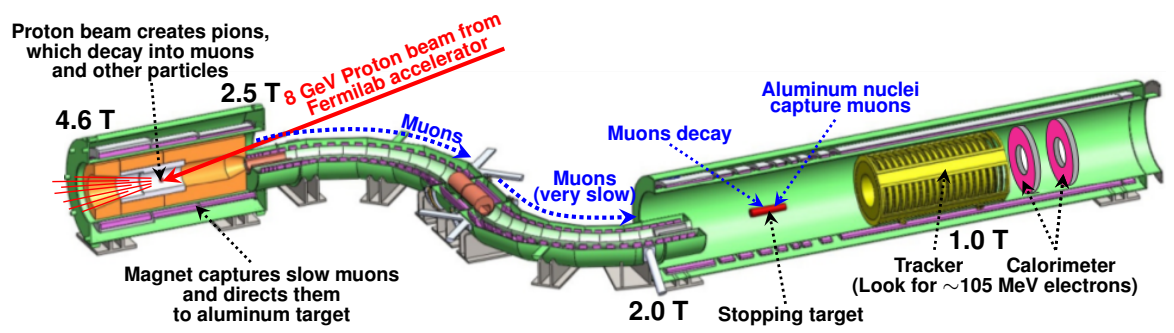

- Graded fields in the 3 solenoid systems are important

- to increase muon yields

- to suppress backgrounds

- to improve geometric acceptance for signal electrons 
TheMhtree Pexpiefthieplpe includes

- Modifications to the accelerator

Pulsed proton beam allows definition of a "Live Window" for the signal to sup press profiptibalenground (1695 ns peak-to-peak):

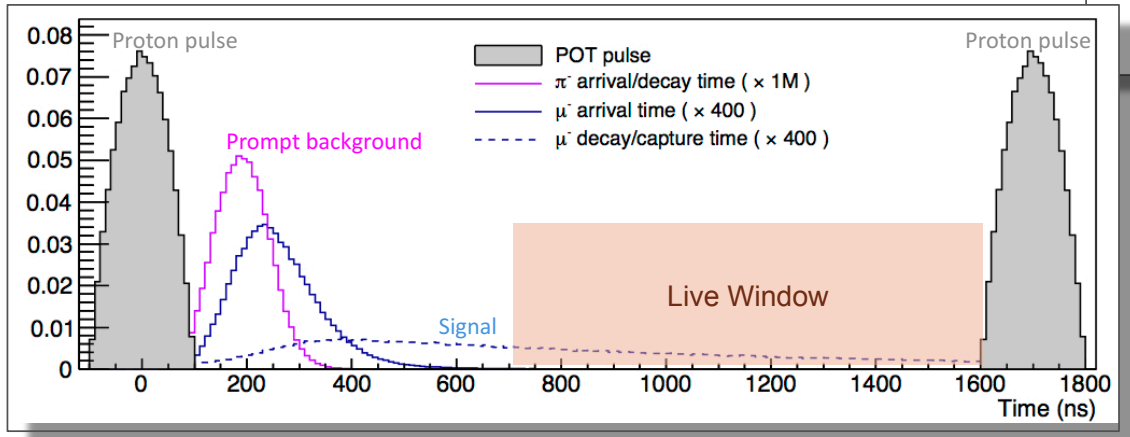

- Fermilab accelerator complex provides optimal pulse spacing for Mu2e Suppress prompt background by many orders of magn mitu

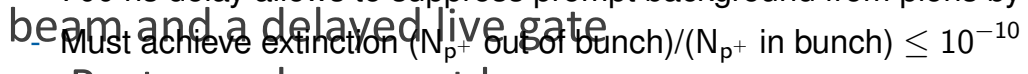

- Proton pulses must be narrow 


\section{Straw drift tube tracker}

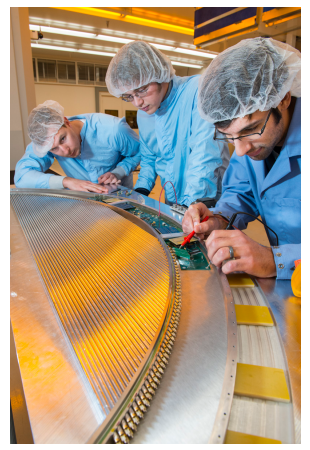

- low mass straw drift tubes (5mm diam.)

- >20000 straws

n in vacuum and at $\sim 1 \mathrm{~T}$ magn. field

- momentum resolution $\sigma_{\mathrm{p}}<180 \mathrm{keV} / \mathrm{c}$

- inner $38 \mathrm{~cm}$ not instrumented $\rightarrow$ "blind" to low-momenta DIO electrons

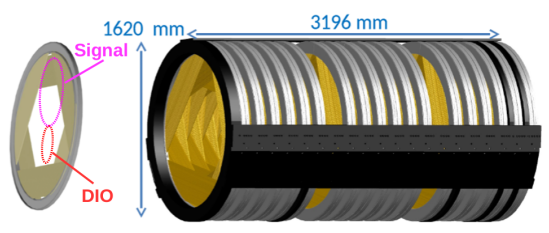




\section{Calorimeter}

- composed of two rings separated by half a wavelength of electron trajectory helix

- each ring composed of $\sim 700$ pure CsI crystals read out by SiPMs

- independent measurement of

- energy $\left(\sigma_{\mathrm{E}} / \mathrm{E} \sim 5 \%\right)$

- time $\left(\sigma_{\mathrm{t}} \sim 0.5 \mathrm{~ns}\right)$

- position $\left(\sigma_{\mathrm{Pos}} \sim 1 \mathrm{~cm}\right)$

- independent trigger information

n particle ID

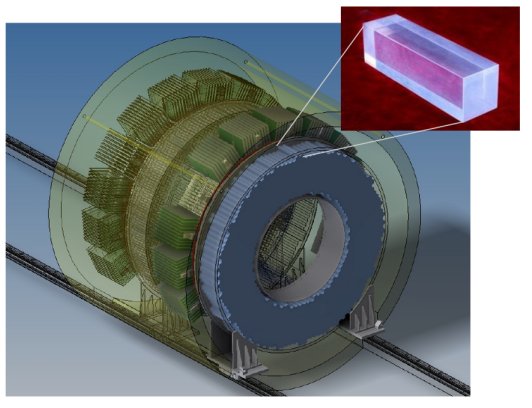




\section{The cosmic ray veto detector}

The cosmic ray veto system (CRV) covers entire Detector solenoid and half of the Transportation Solenoid

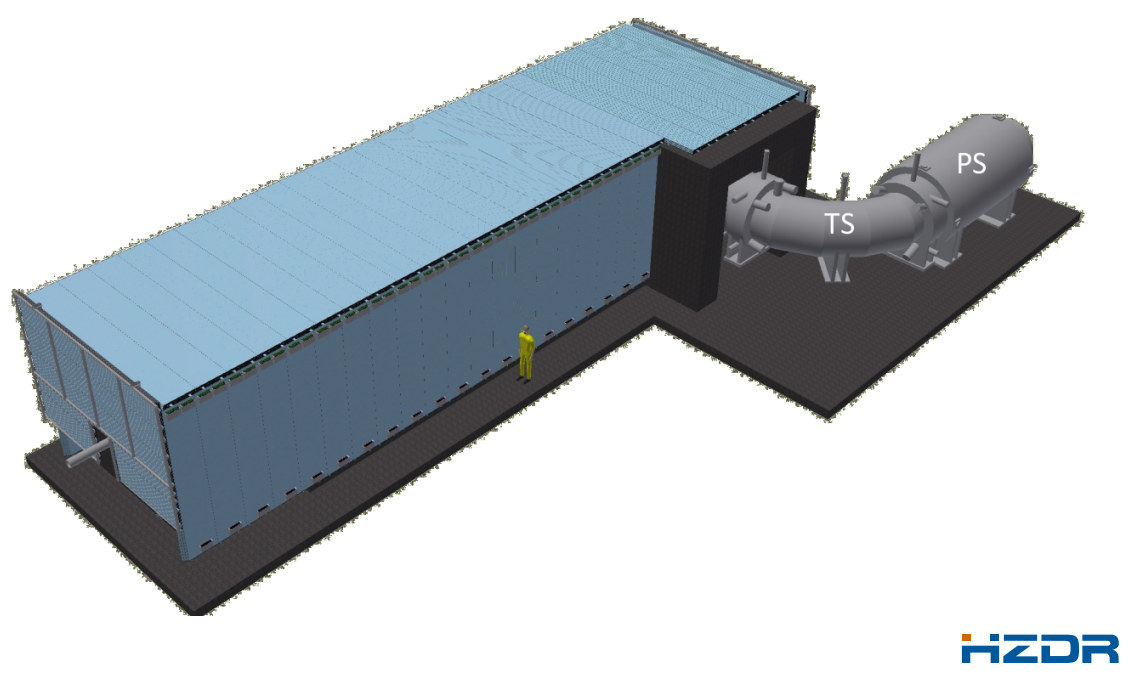




\section{The cosmic ray veto detector}

Without CRV, $\sim 1$ cosmic-ray induced background event per day
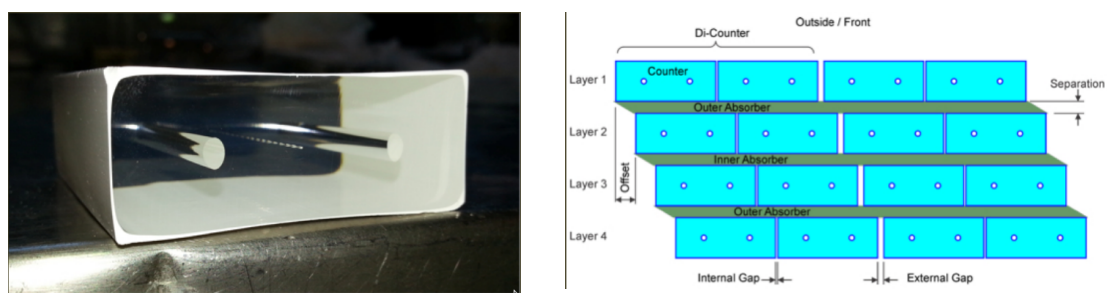

- 4 overlapping layers of scintillator bars $\left(5 \times 2 \times \sim 450 \mathrm{~cm}^{3}\right)$

- 2 wavelength-shifting fibers/bar

- Read out both end of each fiber with SiPMs

- $\epsilon>99.4 \%$ (per layer) achieved in test beam 


\section{The Stopping-Target Monitor}

High-purity Germanium (HPGe) detector to determine overall muon-capture rate on $\mathrm{Al}$ to about the $10 \%$ level

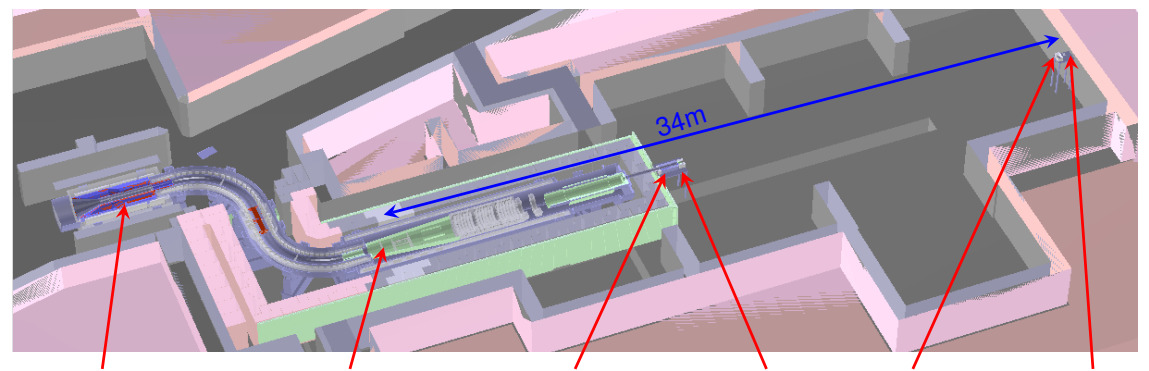

\section{Production Target Stopping Target Sweeper magnet Collimator Collimator HPGe det.}

- measures $X$ - and $\gamma$-rays from muonic Aluminum

- $347 \mathrm{keV} 2 \mathrm{p}-1 \mathrm{~s}$ X-ray ( $80 \%$ of muon stops)

- $844 \mathrm{keV}$ delayed $\gamma$-ray (5\% of muon stops)

- 1809 keV $\gamma$-ray (30\% of muon stops)
- line-of-sight view of Muon Stopping Target

- sweeper magnet to reduce charged particle background and radiation damage to detector 


\section{Magnet production}

In total $75 \mathrm{~km}$ of conductor:
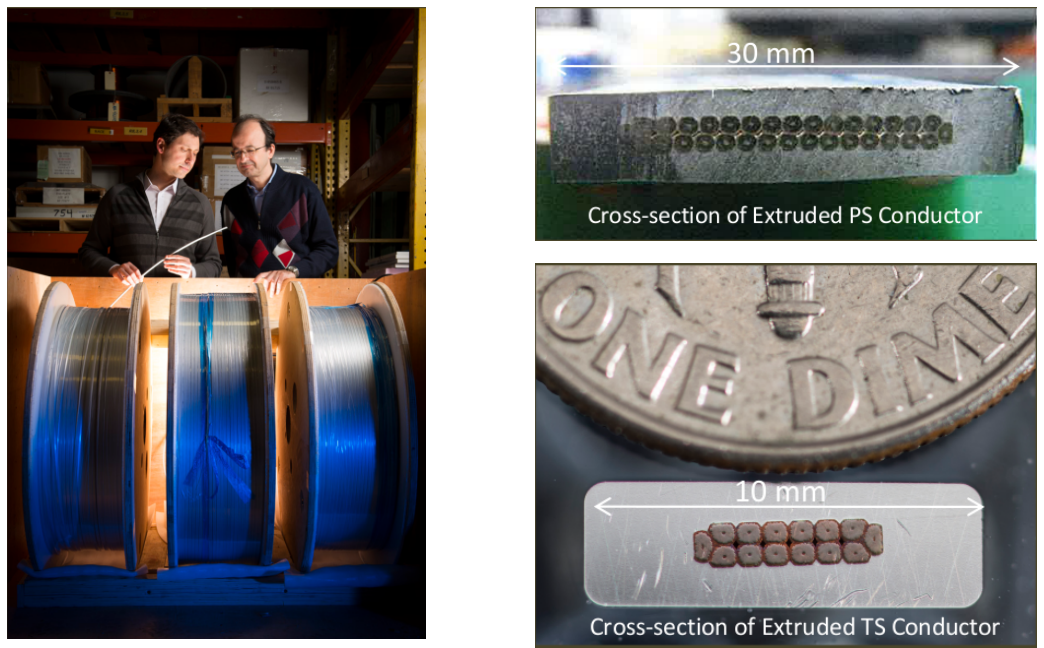

- Conductor production is complete

$$
\text { i元口及 }
$$




\section{Magnet production}

Transport solenoid production at ASG (Genova) and Fermilab:
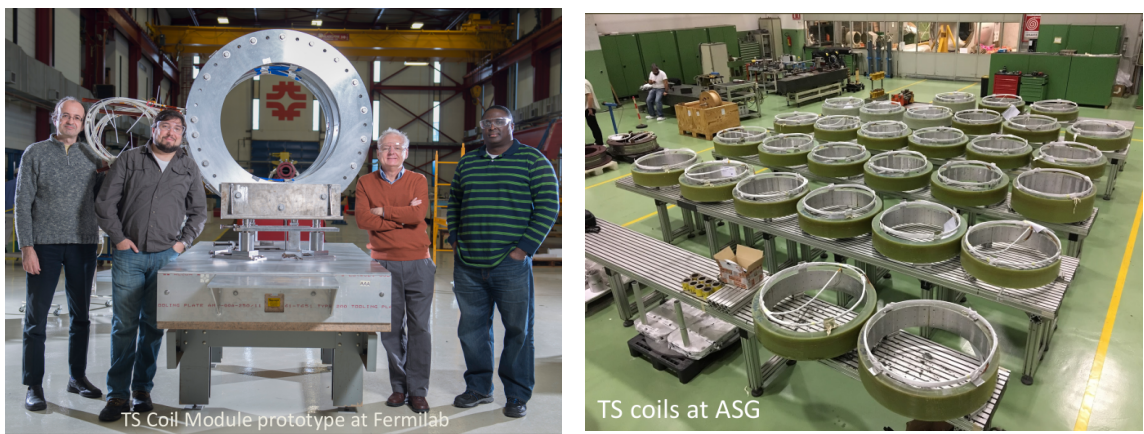

- First TS module at Fermilab undergoing cold test

- All coils have been wound 


\section{Magnet production}

Production and Detector Solenoid production at General Atomics (Tupelo):

n First demonstration coil with two layers of 70 turns each was successfully completed

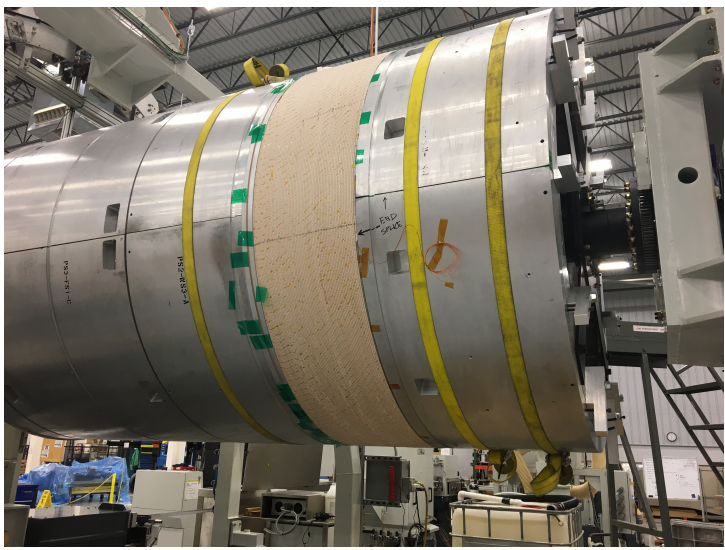




\section{Mu2e@HZDR: The ELBE radiation source}

The ELBE "Electron Linac for beams with high Brilliance and low Emittance" delivers multiple secondary beams.

- $\mathrm{E}_{\mathrm{e}} \leq 40 \mathrm{MeV} ; \mathrm{I}_{\mathrm{e}} \leq 1 \mathrm{~mA}$; Micropulse duration $10 \mathrm{ps}<\Delta \mathrm{t}<1 \mu \mathrm{s}$

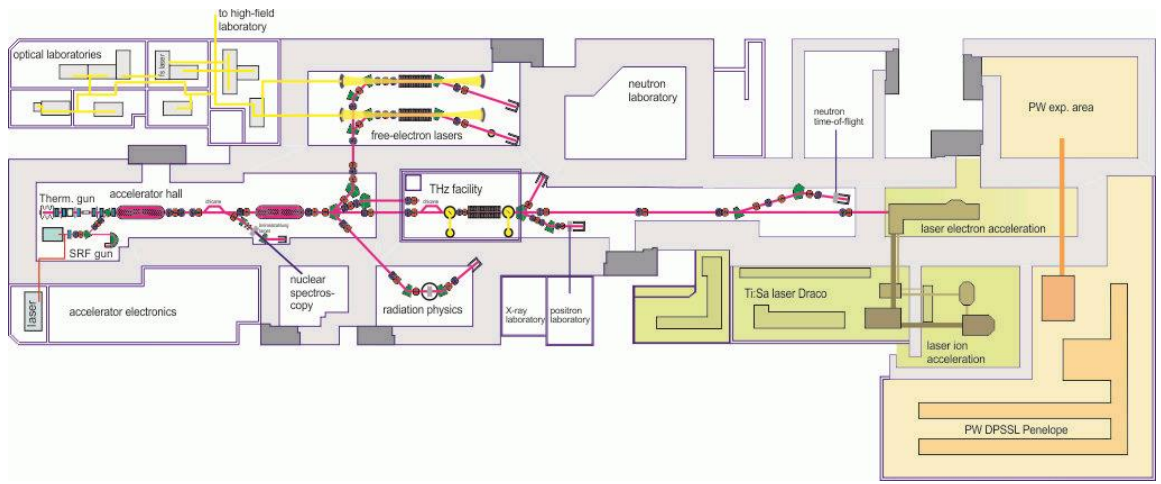




\section{Mu2e@HZDR: The ELBE radiation source}

The ELBE "Electron Linac for beams with high Brilliance and low Emittance" delivers multiple secondary beams.

- $\mathrm{E}_{\mathrm{e}} \leq 40 \mathrm{MeV} ; \mathrm{I}_{\mathrm{e}} \leq 1 \mathrm{~mA}$; Micropulse duration $10 \mathrm{ps}<\Delta \mathrm{t}<1 \mu \mathrm{s}$

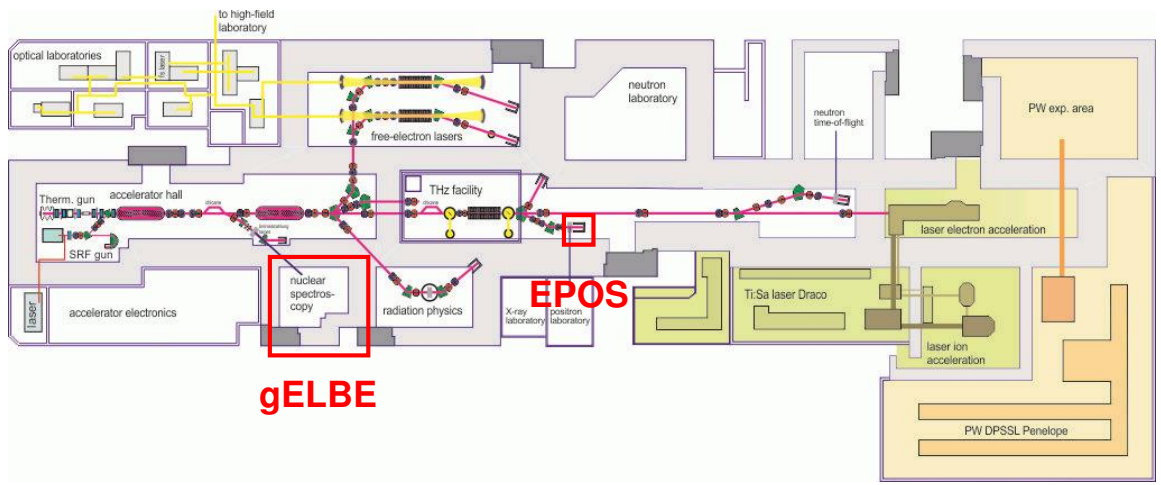

EPOS: Positron (+ Photoneutron) source (Radiation hardness tests) gELBE: Gamma beam facility (HPGe detector design for STM and calorimeter board testing) 


\section{Testing radiation hardness of SiPMs at EPOS}

Positron production by ELBE $30 \mathrm{MeV}$ electron beam on tungsten target is accompanied by a large amount of photoproduced neutrons with an energy spectrum which peaks at $\sim 1 \mathrm{MeV}$.

$\rightarrow$ this matches the expected radiation conditions at Mu2e

- expected neutron fluence has been simulated using FLUKA

- SiPMs from 3 suppliers have been installed on top of the EPOS target bunker for a parasitic beamtime

- dark current of SiPMs has been monitored (stabilized at $20^{\circ} \mathrm{C}$ )

- integrated fluence of more than $8 \times 10^{11} 1$-MeV-equiv. neutrons $/ \mathrm{cm}^{2}$ has been accumulated

- Routinely parasitic irradiation of SiPMs
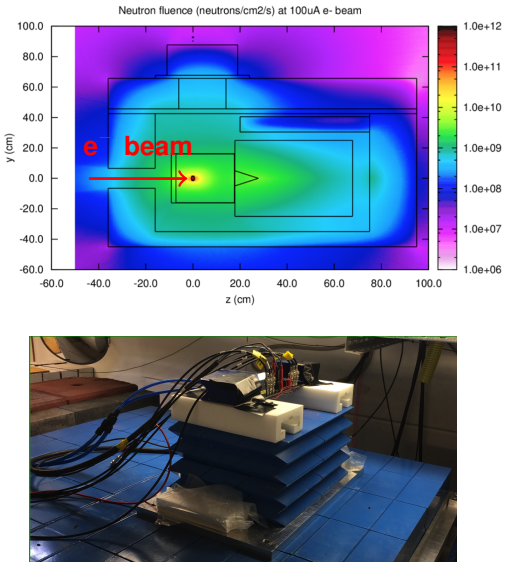


\section{Testing radiation hardness of SiPMs at EPOS}

Positron production by ELBE $30 \mathrm{MeV}$ electron beam on tungsten target is accompanied by a large amount of photoproduced neutrons with an energy spectrum which peaks at $\sim 1 \mathrm{MeV}$.

$\rightarrow$ this matches the expected radiation conditions at Mu2e

- expected neutron fluence has been simulated using FLUKA

- SiPMs from 3 suppliers have been installed on top of the EPOS target bunker for a parasitic beamtime

- dark current of SiPMs has been monitored (stabilized at $20^{\circ} \mathrm{C}$ )

- integrated fluence of more than $8 \times 10^{11} 1$-MeV-equiv. neutrons $/ \mathrm{cm}^{2}$ has been accumulated

- Routinely parasitic irradiation of SiPMs
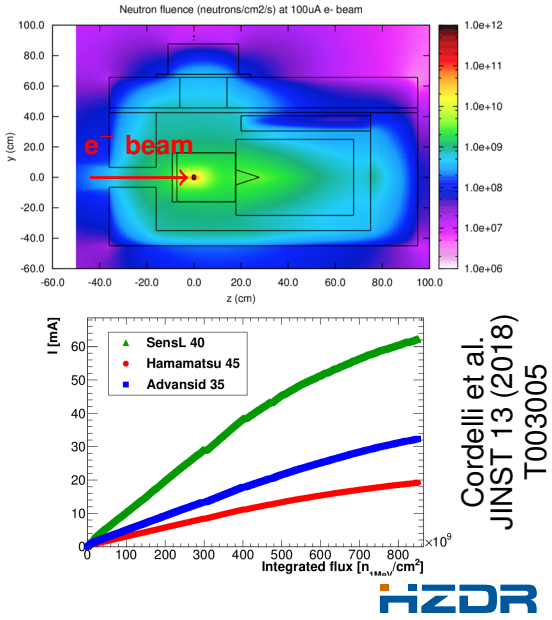


\section{Studying HPGe detector response at gELBE}

The gELBE bremsstrahlung facility was used to study HPGe detector performance. gELBE utilizes Bremsstrahlung production from an electron beam impinging on niobium radiator foils.

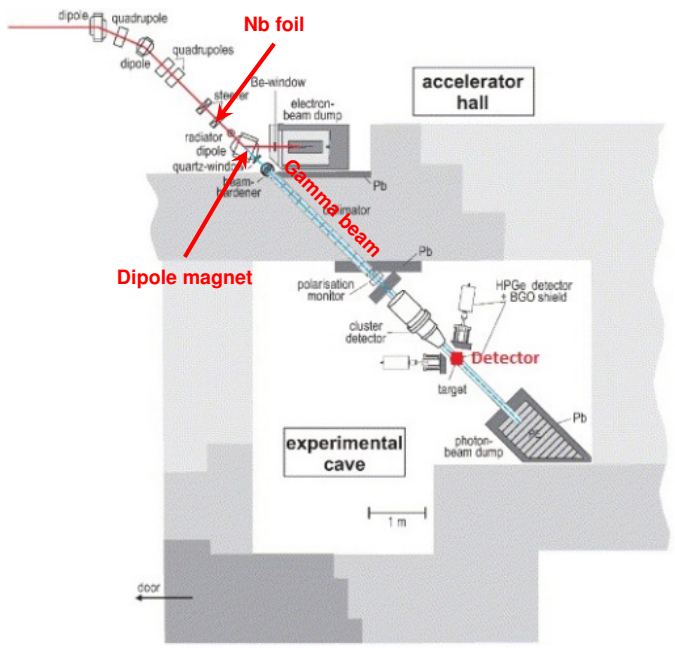




\section{Studying HPGe detector response at gELBE}

gELBE delivers a pulsed $\gamma$-beam with max. energy of $15 \mathrm{MeV}$.

- Up to $125 \mathrm{kHz}$ of gamma rates expected for Mu2e Stopping-Target Monitor HPGe detector during beam pulse

- high average $\gamma$ energy $(\sim 5 \mathrm{MeV})$

- high beam pulse occupancy ( 20\%)

- gELBE pulse separation of $2.4 \mu$ s close to Mu2e's $1.7 \mu$ s proton pulse separation

- Goals of the beamtime:

- Measure HPGe detector performance in the gELBE beam (energy resolution, radiation damage,...)

- Understand best beam and detector geometry and position (including absorbers)

- HZDR provides radiation transport simulations using the FLUKA code to estimate $\gamma$ energy spectrum, energy deposit in crystal etc.

- Detector specifications have been finalized and order has been placed 


\section{Studying HPGe detector response at gELBE}

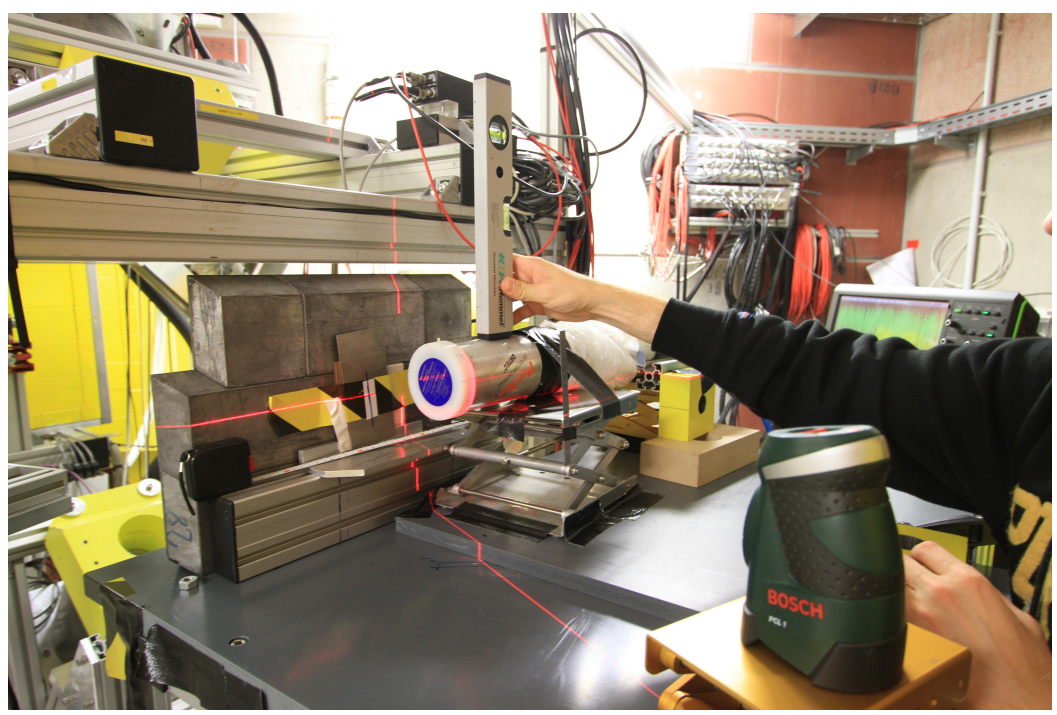




\section{Studying HPGe detector response at gELBE}

Studying energy deposition in crystal:

- Simulate gELBE bremsstrahlung spectrum starting from electron beam hitting niobium foil and propagate it till HPGe detector position

- HPGe detector behind lead wall with $1 \mathrm{~cm}^{2}$ collimator hole and copper/aluminum absorber plates to shield from lead fluorescence.

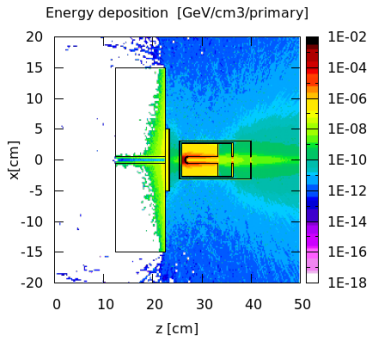

Average energy deposition

$(508.68 \pm 0.11) \mathrm{keV}$

per primary $\gamma$

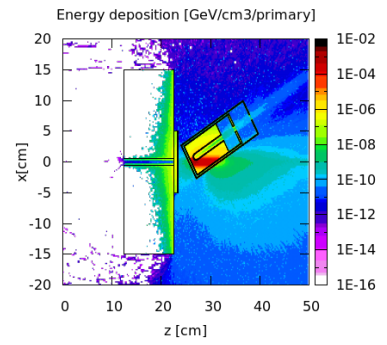

Average energy deposition

$(1846.3 \pm 0.16) \mathrm{keV}$ per primary $\gamma$

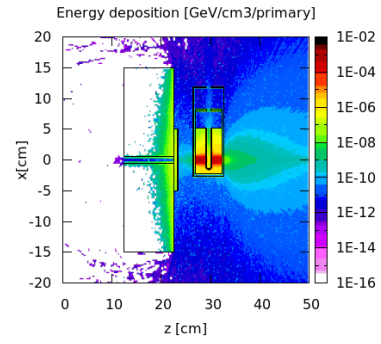

Average energy deposition

(1759.4 \pm 0.08$) \mathrm{keV}$ per primary $\gamma$ 


\section{Irradiation of calorimeter digitizer board at gELBE}

Digitizer board behind lead and PE collimator walls to allow individual irradiation of board components

- Gamma radiation produced by $15 \mathrm{MeV}$ electron beam with $700 \mu \mathrm{A}$ on niobium radiator

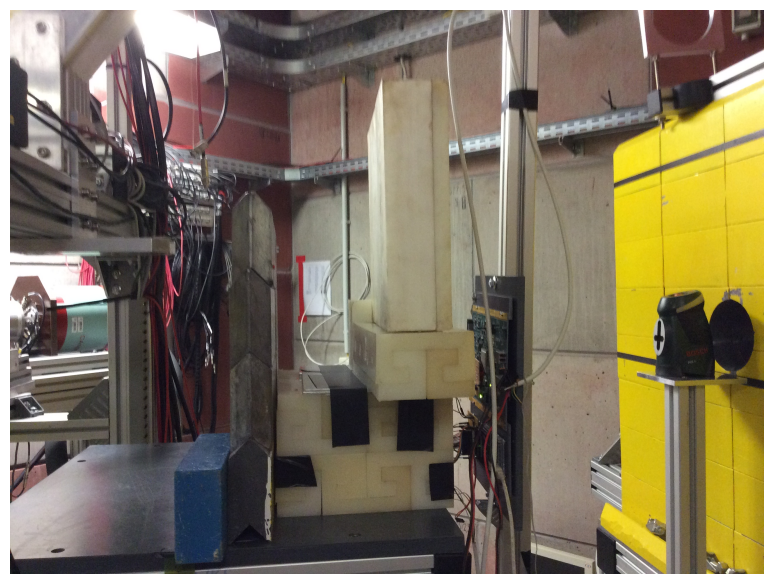




\section{Mu2e MC simulation with FLUKA}

Mu2e Production Target modeled with the FLUKA radiation transport software

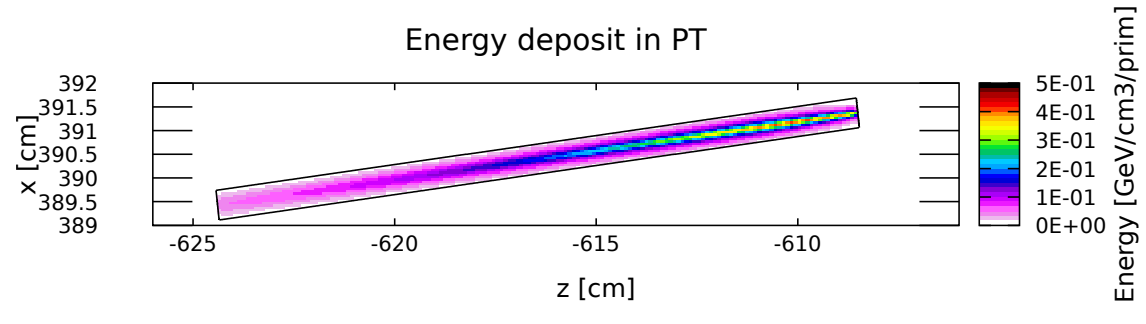




\section{Mu2e MC simulation with FLUKA}

Mu2e Production Target modeled with the FLUKA radiation transport software

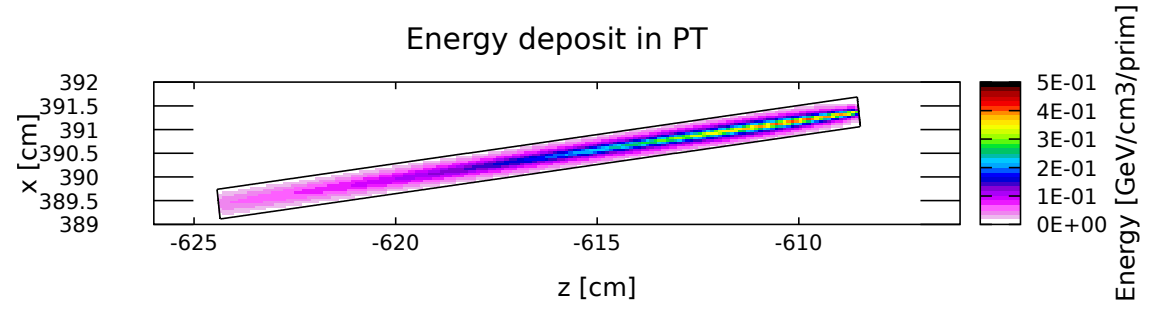

FLUKA finds an average energy deposition of $0.833 \mathrm{GeV} /$ proton \pm $0.2 \%$, which corresponds to 730 Watt@7.3kW beam power. 


\section{Mu2e MC simulation with FLUKA}

Mu2e Production Target modeled with the FLUKA radiation transport software

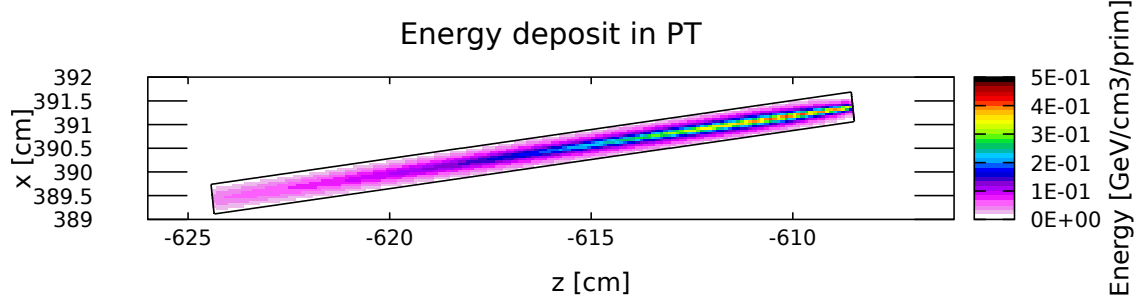

FLUKA finds an average energy deposition of $0.833 \mathrm{GeV} /$ proton \pm $0.2 \%$, which corresponds to 730 Watt@7.3kW beam power.

In good agreement with the results obtained with other Monte Carlo codes:

703 Watt@7.3kW beam power (G4Beamline)

713 Watt@7.3kW beam power (MARS) 


\section{Conclusion \& Outlook}

- The Mu2e experiment at FERMILAB will search for the neutrinoless conversion of a muon into an electron in the coulomb field of an Aluminum nucleus

- projected upper limit: $8 \times 10^{-17}(90 \% \mathrm{CL})$

- Detector design ready, construction started

- Solenoid design ready, coil fabrication started

- HZDR contributes with beamtimes at the ELBE radiation source for tests of radiation hardness of calorimeter components and HPGe detector design for STM

- In addition studies of production and stopping target with FLUKA $\mathrm{MC}$ simulation code are under way

- implement more of the geometry

- include magnetic field

- With physics dataking starting in 2023, Mu2e will either unambiguously discover CLFV or push the limit on muon $\rightarrow$ electron conversion by four orders of magnitude 


\section{Mu2e Collaboration}

More than 200 scientists from 38 institutions:

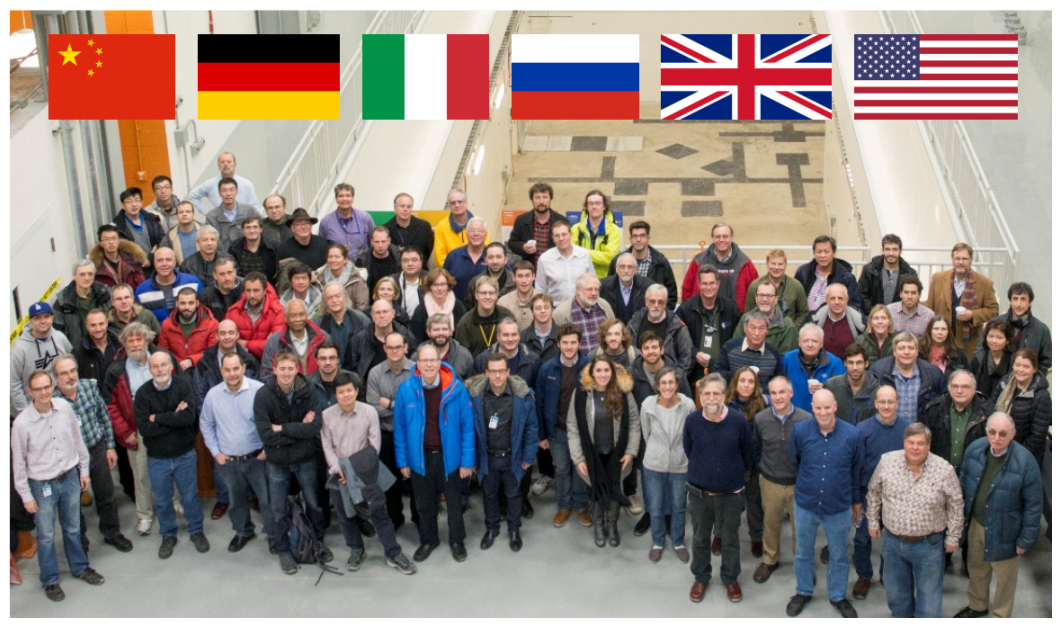

